\title{
Evidence-Based Medicine Research on Prescriptions in Synopsis of the Golden Chamber in the Treatment of Pelvic Inflammatory Disease
}

\author{
Mengkui Li' ${ }^{1}$ Junsheng Song ${ }^{*}$, Rong Shang2, Xishun Pan1 \\ ${ }^{1}$ Tianjin University of Traditional Chinese Medicine, Tianjin, China \\ ${ }^{2}$ First Teaching Hospital of Tianjin University of Traditional Chinese Medicine, Tianjin, China \\ Email: *songjunsheng00@126.com
}

How to cite this paper: Li, M.K., Song, J.S., Shang, R. and Pan, X.S. (2017) Evidence-Based Medicine Research on Prescriptions in Synopsis of the Golden Chamber in the Treatment of Pelvic Inflammatory Disease. Open Journal of Obstetrics and Gynecology, 7, 61-70.

http://dx.doi.org/10.4236/ojog.2017.71007

Received: November 3, 2016

Accepted: December 27, 2016

Published: December 30, 2016

Copyright $\odot 2017$ by authors and Scientific Research Publishing Inc. This work is licensed under the Creative Commons Attribution International License (CC BY 4.0).

http://creativecommons.org/licenses/by/4.0/

\begin{abstract}
Purpose: Based on the National Natural Science Foundation of China, we collect and sort out the literature published in approximately the last 30 years, which use prescriptions in Synopsis of the Golden Chamber to treat pelvic inflammatory disease (PID). This study provides theoretical basis for the treatment of PID; we recommend this achievement to colleagues. Method: Firstly, we searched and collected the literatures from last three decades in CNKI, Wanfang and VIP databases, which are about classical prescriptions treating endometriosis. Then, by screening all the literatures, we obtained the clinical research literatures and individual case reports. Finally, we went through the internal quality of the two categories of literatures to get the dominant prescriptions. Using a retrospective study of evidence-based medicine research, we comprehensively collect literature, standardize the disease spectrum, classify the evidence, and then evaluate the internal quality. Results: We refine 4 prescriptions which commonly are used in the treatment of PID; in other words, they are also the clinical symptoms of PID: Gui Zhi Fu Ling Wan symptom, which manifests blood stasis and cold-damp stagnancy; Dang Gui Shao Yao San symptom, which manifests disharmony of liver and spleen, qi-blood stasis and damp obstruction; Da Huang $\mathrm{Mu}$ Dan Tang symptom, which manifests the gathering toxic heat and qi-blood stasis; Yi Yi Fu Zi Bai Jiang San symptom, which manifests the internal toxic heat and qi-blood injury. Conclusion: This study is gospel for the patients with recurrent episodes of PID who can't often use antibiotics. At the same time, it is also a green therapeutics which Chinese ancients dedicated to human medicine.
\end{abstract}

\section{Keywords}

Traditional Chinese Medicine, Evidence-Based Medicine, Quality of Evidence, Prescriptions in Synopsis of Golden Chamber, The Treatment of Pelvic Inflammatory Disease 


\section{Introduction}

\subsection{Understanding of Pelvic Inflammatory Disease in Modern Medicine}

Pelvic inflammatory disease (PID) refers to the inflammation of the female genital tract and surrounding tissue, mainly including endometritis, salpingitis, tubal ovarian abscess and pelvic peritonitis [1]. It can be divided into acute and chronic types according to its pathogenesis process and clinical manifestation.

Acute PID has a history of acute infection; patients may experience faint pain in the lower abdomen, muscle tension, tenderness and rebound tenderness, with a fast heart rate, fever, a large quantity of purulent secretion from the vagina. When seriously ill, patients will have a high fever, headache, chills, anorexia, a lot of odiferous yellow leukorrhea, distending pain and tenderness in the lower abdomen, aching pain in the loin, etc. When experiencing peritonitis, there will be nausea, abdominal distension, vomiting, diarrhea, etc. When there is a formation of pus, patients may have a symptom of lower abdominal mass and local oppression to stimulation, and also have difficulty urinating, frequent micturition, odynuria with the mass in front. The mass located in the rear can cause diarrhea.

The chronic PID patients are susceptible to fatigue and sometimes have a low fever. Owing to the long duration, some patients may exhibit symptoms of neurasthenia, such as insomnia, lack of energy, and general malaise. Lower abdominal bulge and pain, aching pain in the loin and sacrum often take a turn for deteriorate with tiredness, after sexual intercourse and around the menstruation. Chronic inflammation may cause pelvic cavity thrombosis; menoxenia; menstrual disorders may occur if the ovary function is damaged; tubal adhesion blocking will lead to infertility.

Principles of treatment of this disease are: 1) The acute PID patients should be given active and thorough treatment, in order to prevent the inflammation becoming chronic; the latter is more stubborn, and will affect the reproductive function. 2) Pathogen treatment. Most patients of PID are mixed infections; if the bacterial culture is positive, we can choose the most effective antibiotic for treatment depending on the drug sensitive test. 3) For patients with inflammatory mass, we should consider surgery immediately if treatment with antibiotics is not effective.

\subsection{Understanding of Pelvic Inflammatory Disease in Traditional Chinese Medicine}

PID belongs in the field of abdominal pain, leukorrhagia, abdominal mass, irregular menstruation, and sterility in traditional Chinese medicine. Kidney deficiency with disharmony of qi and blood, and stasis of damp-heat are the most common pathogenesis, eventually leading to blockage of the uterine collaterals, obstruction of the meridians, not general pain. The appropriate therapies are mainly nourishing the kidney and harmonizing qi and blood, resolving dampness and alleviating fever, activating blood to unblock collaterals.

\subsection{The Framework and Value of Synopsis of the Golden Chamber}

Synopsis of the Golden Chamber, as a section of Treatise on Febrile and Miscellaneous Diseases, composed by Zhang Zhongjing in the late age of East Han Dynasty, is existing 
and the earliest book about discussing clinical miscellaneous diseases in China. The book records three articles about gynecological diseases, has very high guiding significance and practical value in the development of traditional Chinese Medicine in later clinical medicine, and has made a significant contribution to and had far-reaching influence on the development of clinical medicine. Therefore, focus on researching the literature which use classical prescriptions to treat PID in nearly 30 years, we use the method of evidence-based medicine, and finally obtain the most effective prescriptions and clinical classification. This study will provide theoretical basis in the treatment of PID.

\subsection{Prescriptions and Quality Level in Clinical Research Literatures}

Evidence-based medicine commonly refer to GRADE standard to evaluate quality of literatures, whereas Evidence-based medicine research in Traditional Chinese Medicine cannot ponderously apply the exact same items. Consequently, the following quality level identification is based on a modified GRADE standard that was discussed and recognized by several rounds of professionals, adding four items of the standard which are: addition or subtraction of prescriptions, the original does and composition of the prescription, using Shanghan Zabing Lun prescriptions only and whether researches and cases are from before the year of 1979. The rest of items are the same as in GRADE standard [2]. Detailed information is shown in Table 1.

\section{Materials and Methods}

\subsection{The Overview of Prescriptions in Synopsis of the Golden Chamber in Treatment of PID}

We collect and sort out the literature published in approximately the last 30 years, which use the prescriptions in Synopsis of the Golden Chamber to treat PID, and then standardize the disease spectrum, classify the evidence, and evaluate the internal quality. Finally we refine 19 prescriptions which commonly are used in the treatment of PID, among them 10 prescriptions have been carried out in clinical studies, 18 prescriptions have been reported as case experience. The literature frequencies of each prescription are shown in Table 2 and Table 3.

Table 1. Deciding factors in quality level.

\begin{tabular}{lc}
\hline Influence of orientation & Specific factors \\
\hline Degrading factors & $\begin{array}{c}\text { Research limitation } \\
\text { Inconsistent results } \\
\text { Indirect evidence } \\
\text { Low accuracy } \\
\text { Publication bias }\end{array}$ \\
Upgrading factors & $\begin{array}{c}\text { Addition or subtraction of prescriptions } \\
\text { Original does \& composition of the prescription } \\
\text { Using shanghan zabing lun prescriptions only } \\
\text { Researches and cases from before year of 1979 } \\
\text { High effective value } \\
\text { Miscellaneous factors reducing clinical effect } \\
\text { Does-effect relationship }\end{array}$ \\
\hline
\end{tabular}


Table 2. Literature prescription spectrum of clinical research of PID.

\begin{tabular}{cccccc}
\hline & Prescription name & Frequency & & Prescription name & Frequency \\
\hline 1 & Gui Zhi Fu Ling Wan & 48 & 6 & Da Huang Fu Zi Tang & 1 \\
2 & Dang Gui Shao Yao San & 27 & 7 & Da Huang Zhe Chong Wan & 1 \\
3 & Da Huang Mu Dan Tang & 23 & 8 & Huang Qi Jian Zhong Tang & 1 \\
4 & Yi Yi Fu Zi Bai Jiang San & 11 & 9 & Ba Wei Di Huang Wan & 1 \\
5 & Xia Yu Xue Tang & 2 & 10 & Wen Jing Tang & 1 \\
\hline
\end{tabular}

Table 3. Literature prescription spectrum of case experience of PID.

\begin{tabular}{cccccc}
\hline & Prescription name & Frequency & & Prescription name & Frequency \\
\hline 1 & Dang Gui Shao Yao San & 34 & 6 & Da Huang Zhe Chong Wan & 2 \\
2 & Gui Zhi Fu Ling Wan & 21 & 7 & Dang Gui Bei Mu Ku Shen Wan & 2 \\
3 & Da Huang Mu Dan Tang & 11 & 8 & Pai Nong Tang & 2 \\
4 & Yi Yi Fu Zi Bai Jiang San & 7 & 9 & Wen Jing Tang & 2 \\
5 & Huang Qi Jian Zhong Tang & 4 & 10 & Huang Qi Gui Zhi Wu Wu Tang & 1 \\
\hline
\end{tabular}

As can be seen from the table, the clinical research literature mainly focused on Gui Zhi Fu Ling Wan, followed by Dang Gui Shao Yao San, Da Huang Mu Dan Tang and Yi Yi Fu Zi Bai Jiang San; and case experience literature is focused on Dang Gui Shao Yao San, followed by Da Huang Mu Dan Tang and Yi Yi Fu Zi Bai Jiang San, the remaining prescriptions are used less frequently.

\subsection{Evaluation of Clinical Evidence and Quality}

The clinical evidence of PID is from clinical studies and case experience literature, clinical evidence has 116 articles, case experience literature has 82 articles. In clinical research literature, there are 31 randomized controlled trials, 3 quasi-randomized controlled trials, 5 non-randomized controlled trials, 77 case series observations; there are 82 articles in case experience literature, reported 94 proven cases of PID. The quality of clinical research is not high. However, combined with the case history, it fully demonstrates the important role of the prescriptions in the treatment of PID.

\subsubsection{Quality Evaluation of Clinical Research Literature}

\section{1) Gui Zhi Fu Ling Wan}

In the 37 documents there are 1 piece of system evaluation, 20 randomized controlled trials, 3 quasi-randomized controlled trials, 5 non-randomized controlled trials, 8 case series observations. The graded evaluation of evidence quality is shown in Table 4 (low quality is omitted).

\section{2) Dang Gui Shao Yao San}

Totally 24 documents included: 9 randomized controlled trials, 6 non-randomized controlled trials, 9 case series observations. All documents are distributed between 1998-2007. The graded evaluation of quality of evidence is shown in Table 5. As can be seen, there are 2 high quality evidence, 9 medium quality evidence. The main factors for the downgrade evidence are the limitations of study, added drug interference, low 
Table 4. List of evidence quality of clinical research literature of Gui Zhi Fu Ling Wan.

\begin{tabular}{ccccc}
\hline Included studies & $\begin{array}{c}\text { Publishing } \\
\text { time }\end{array}$ & $\begin{array}{c}\text { Document } \\
\text { type }\end{array}$ & Evidence lifting factor & Grade \\
\hline Yanxia Liu [3] & 2006 & CR & $\begin{array}{c}\text { Dose-response relationship (+1) } \\
\text { Zhongjing prescription (+1) single } \\
\text { intervention of Zhongjing prescription (+1) }\end{array}$ & High \\
Jianxin Zhao [4] & 2006 & 'RCT & $\begin{array}{c}\text { Limitations of the study (-2) dose-response } \\
\text { relationship (+1) Zhongjing prescription (+1) }\end{array}$ & High \\
Huiying Yang [5] & 2007 & CR & $\begin{array}{c}\text { Zhongjing prescription (+1) single } \\
\text { intervention of Zhongjing prescription (+1) }\end{array}$ & High \\
Xiufen Li [6] & 2008 & 'RCT & $\begin{array}{c}\text { Limitations of the study (-2) Zhongjing } \\
\text { prescription (+1) single intervention of }\end{array}$ & High \\
Yingjie Li [7] & 2009 & CR & $\begin{array}{c}\text { Zhongjing prescription (+1) } \\
\text { intervention of Zhongjing prescription (+1) }\end{array}$ & High \\
Xinling Wang [8] & 2000 & CR & Zhongjing prescription (+1) & Medium \\
Qiao Lu [9] & 2004 & CR & Zhongjing prescription (+1) & Medium \\
Guozhen Chen [10] & 2005 & CR & Zhongjing prescription (+1) & Medium \\
\hline
\end{tabular}

Table 5. List of evidence quality of clinical research literature of Dang Gui Shao Yao San.

\begin{tabular}{ccccc}
\hline Included studies & $\begin{array}{c}\text { Publishing } \\
\text { time }\end{array}$ & $\begin{array}{c}\text { Document } \\
\text { type }\end{array}$ & Evidence lifting factor & Grade \\
\hline Hong Zhang [1] & 2009 & CR & $\begin{array}{c}\text { Zhongjing prescription (+1) single } \\
\text { intervention of Zhongjing prescription (+1) }\end{array}$ & High \\
Hong Zhang [12] & 2009 & RCT & $\begin{array}{c}\text { Low accuracy (-1) dose-response relationship } \\
(+1) \text { Zhongjing prescription (+1) single } \\
\text { intervention of Zhongjing prescription (+1) }\end{array}$ & High \\
Chunxian Zhang [13] & 2006 & CR & Zhongjing prescription (+1) & Medium \\
Yunzhong Lin [14] & 2006 & CR & Zhongjing prescription (+1) & Medium \\
Xiaomei Ma [15] & 2008 & CR & Zhongjing prescription (+1) & Medium \\
\hline
\end{tabular}

accuracy. Evidence upgrade factor is mainly using single intervention of Zhongjing prescription.

\section{3) Da Huang Mu Dan Tang}

A total of 13 documents included: 5 randomized controlled trials, 1 quasi-randomized controlled trials, 7 case series observation. All documents are distributed between 1994-2007. The graded evaluation of evidence quality is shown in Table 6. As can be seen, there are 1 high quality evidence, 3 medium quality evidence, the rest of quality evidence is low. The main factors for the downgrade of evidence are the limitations of the study, added drug interference, low accuracy. Evidence upgrade factor is mainly using Zhongjing prescription and single intervention of Zhongjing prescription.

\section{4) Yi Yi Fu Zi Bai Jiang San}

Totally 10 documents included: 8 randomized controlled trials, 2 case series observations. All documents are distributed between 1993-2007. The graded evaluation of evidence quality is shown in Table 7 . The research and quality of this prescription is very low. 
Table 6. List of evidence quality of clinical research literature of Da Huang Mu Dan Tang.

\begin{tabular}{ccccc}
\hline Included studies & $\begin{array}{c}\text { Publishing } \\
\text { time }\end{array}$ & $\begin{array}{c}\text { Document } \\
\text { type }\end{array}$ & Evidence lifting factor & Grade \\
\hline Hanmin Xu [16] & 1992 & CR & $\begin{array}{c}\text { Zhongjing prescription (+1) single } \\
\text { intervention of Zhongjing prescription (+1) }\end{array}$ & High \\
Lina Zhang [17] & 1999 & CR & $\begin{array}{c}\text { Dose-response relationship (+1) } \\
\text { Zhongjing prescription (+1) }\end{array}$ & Medium \\
Yuxia Wang [18] & 1999 & CR & $\begin{array}{c}\text { Limitations of the study (-2) } \\
\text { Zhongjing prescription (+1) }\end{array}$ & Medium \\
Defang Hao [19] & 2008 & 'RCT & \begin{tabular}{l} 
Zhong \\
\hline
\end{tabular}
\end{tabular}

Table 7. List of evidence quality of clinical research literature of Yi Yi Fu Zi Bai Jiang San.

\begin{tabular}{ccccc}
\hline Included studies & Publishing time & Document type & Evidence lifting factor & Grade \\
\hline Fushan Qin [20] & 2007 & CR & Nothing & Low \\
Tao Zhang [21] & 1993 & CR & Added drug interference (-1) & Very low \\
Huaisheng Li [22] & 1996 & CR & Added drug interference (-1) & Very low \\
Chonglin Mou [23] & 1997 & CR & Added drug interference (-1) & Very low \\
Yan Shi [24] & 2001 & CR & Added drug interference (-1) & Very low \\
Xiaofen Wei [25] & 2002 & CR & Added drug interference (-1) & Very low \\
Weiping Shen [26] & 2003 & CR & Added drug interference (-1) & Very low \\
Yufang He [27] & 2005 & 'RCT & Limitation of the study (-2) & Very low \\
& & & Added drug interference (-1) & \\
Yijuan Zhang [28] & 2006 & CR & Added drug interference (-1) & Very low \\
Jianchun Yu [29] & 2008 & CR & Added drug interference (-1) & Very low \\
\hline
\end{tabular}

\subsubsection{Case Experience Literature}

Totally there are 94 case histories included, using Dang-gui-shao-yao-san, Gui-zhi-fuling-wan, Da-huang-mu-dang-tang,Yi-yi-fu-zi-bai-jiang-san and so on. The date of publication is distributed between 1979-2009. The graded evaluation of evidence quality of each prescription is shown in Table 8. As can be seen, all the related medical records are low quality except the above four Prescriptions, the average quality of which is medium.

\section{Results}

According to existing literature, the common clinical symptoms of PID are Gui Zhi Fu Ling Wan symptom, which manifests blood stasis and cold-damp stagnancy, Dang Gui Shao Yao San symptom, which manifests disharmony of liver and spleen, qi-blood stasis and damp obstruction, Da Huang Mu Dan Tang symptom, which manifests the gathering toxic heat and qi-blood stasis, Yi Yi Fu Zi Bai Jiang San symptom, which manifests the internal toxic heat and qi-blood injury (see Figure 1).

\section{Discussion}

According to the literature report, we can draw conclusions on the basis of evidence- 
Gui Zhi Fu Ling Wan

High quality evidence

High frequency symptom

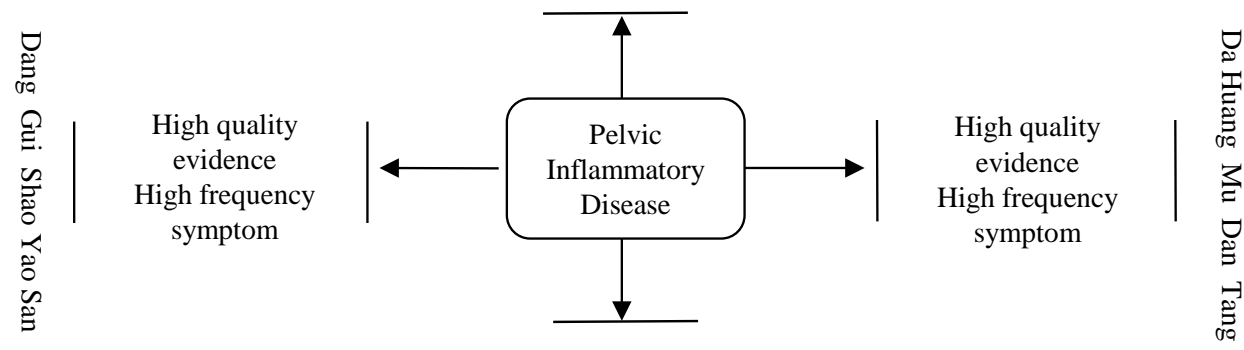

Low quality evidence

High frequency symptom

Yi Yi Fu Zi Bai Jiang San

Figure 1. Rule of pelvic inflammation evidence.

Table 8. List of evidence quality of case experience documentary.

\begin{tabular}{ccccc}
\hline Prescription name & Publishing time & Number of case history & Average quality score & Grade \\
\hline Dang Gui Shao Yao San & $1979-2009$ & 34 & 47.01 & medium \\
Gui Zhi Fu Ling Wan & $1979-2007$ & 21 & 49.37 & medium \\
Da Huang Mu Dang Tang & $1985-2008$ & 11 & 47.53 & medium \\
Yi Yi Fu Zi Bai Jiang San & $1987-2008$ & 7 & 48.44 & medium \\
Da Huang Fu Zi Tang & 1990 & 1 & 37.14 & low \\
Chi Dou Dang Gui San & 2006 & 1 & 38.94 & low \\
\hline
\end{tabular}

based medicine research: Gui Zhi Fu Ling Wan, a total of 48 articles, 3993 cases included; followed by Dang Gui Shao Yao San, a total of 27 articles, 2241 cases included; Dai Huang Mu Dan Tang, a total of 23 articles, 3076 cases included; Yi Yi Fu Zi Bai Jiang San, a total of 11 articles, 1012 cases included. High-quality evidence is distributed in Gui Zhi Fu Ling Wan, Dang Gui Shao Yao San and Dai Huang Mu Dan Tang, the other prescriptions are medium and low-quality evidence. It can be seen that although the prescription types have a wide distribution, they both have a certain clustering irrespective of the literature frequency or the evidence quality.

\subsection{Gui Zhi Fu Ling Wan}

In the chapter on Pregnancy Disease, Gui Zhi Fu Ling Wan is the most important prescription in the treatment of blood stasis and stagnancy of cold-damp, the main symptom manifests as abnormal changes in menstruation quantity and incessant uterine bleeding. However, there is no related symptoms discourse on treatment of PID. The method of treating this disease should be dispelling stasis, the disease will recover as the stasis is cleared up. The prescription is composed of Gui Zhi, Fu Ling, Mu Dan Pi, Tao Ren, Shao Yao. Pelvic inflammation is one of the most common conditions within the disease spectrum of this prescription. High-quality evidence shows that Gui Zhi Fu Ling Wan has curative effect in clinical practice. Thus it can be seen that one of the 
common clinical pathogenesis of this disease is blood stasis and cold-damp stagnation, many people suffer from this condition.

\subsection{Dang Gui Shao Yao San}

In the chapter on Pregnancy Disease, Dang Gui Shao Yao San is the most important prescription in the treatment of disharmony of liver and spleen and abdominal pain caused by qi-blood stasis and damp obstruction. The main symptom is abdominal pain. The prescription is composed of Dang Gui, Shao Yao, Fu Ling, Bai Zhu, Ze Xie, Chuan Xiong. Pelvic inflammation is one of the most common conditions within the disease spectrum of this prescription. High-quality evidence shows that one of the common clinical pathogenesis of this disease is disharmony of liver and spleen, qi-blood stasis and damp obstruction, many people suffer from this condition.

\subsection{Da Huang Mu Dan Tang}

In the chapter on sores, carbuncles, intestinal carbuncles and spreading effusive sores, Da Huang Mu Dan Tang is mainly used to treat acute appendicitis where pus has not yet developed. The main symptom manifests as swelling and hardening in the lower abdomen, causing aching upon pressure and patients refuse to be pressed. The prescription is composed of Da Huang, Mu Dan Pi, Tao Ren, Dong Gua Zi, Mang Xiao. Pelvic inflammation is one of the most common conditions within the disease spectrum of this prescription. High-quality evidence shows that to hot compress or oral Da Huang Mu Dan Tang has a curative effect in clinical practice. Thus it can be seen that one of clinical common pathogenesis of the disease is the gathering toxic heat and qiblood stasis, many people suffer from this condition.

\subsection{Yi Yi Fu Zi Bai Jiang San}

In the chapter on sores, carbuncles, intestinal carbuncles and spreading effusive sores, Yi Yi Fu Zi Bai Jiang San is mainly used to treat developed pus, where the main symptom manifests as abdominal pain and scaly dry skin. The prescription is composed of Yi Yi Ren, Fu Zi, Bai Jiang Cao. Pelvic inflammation is one of the most common conditions within the disease spectrum of this prescription. Low-quality evidence shows that flavored Yi Yi Fu Zi Bai Jiang San has a curative effect in clinical practice. Thus it can be seen that one of the common clinical pathogenesis of this disease is internal toxic heat and qi-blood injury. Although the supporting evidence is low intensity, the prescription can also be taken into consideration in clinical practice when needed.

\section{Conclusion}

Through the evidence-based medicine research and evidence quality evaluation, it can be seen that there is a tendency to treat PID with prescriptions in Synopsis of the Golden Chamber. Thus symptoms of Gui Zhi Fu Ling Wan, Dang Gui Shao Yao San, Da Huang Mu Dan Tang and Yi Yi Fu Zi Bai Jiang San are likely to be the major symptoms of PID in modern clinical environment. This study is gospel for the patients with recurrent episodes of PID who can't often use antibiotics. At the same time, it is also a green therapeutics which Chinese ancients dedicated to human medicine. 


\section{Acknowledgements}

This Project was supported by grants from the National Natural Sciences Foundation of China: Establish Shanghan Lun Prescriptions Treating Chronic Diseases Evidencebased Decision-making Research Based on Multi-level Evidence Fusion Theory (No. 81373891).

\section{References}

[1] Ren, M.L. (2008) Physician's Handbook of Obstetric and Gynecology. Anhui Science \& Technology Press, Hefei, 320.

[2] Song, J.S. (2012) Research on Laws of Shang Han Lun Prescriptions Treating Frequent Diseases and Syndromes. China Press of Traditional Chinese Medicine, Beijing, 13-14.

[3] Liu, Y.X. and Li, X.R. (2006) Guizhi Fuling Capsule for the Treatment of Chronic Pelvic Inflammatory Disease, 150 Cases of Clinical Analysis. Chinese Community Doctors Comprehensive Edition, 8, 119.

[4] Zhao, J.X., Han, J., Zhu, B.J., et al. (2006) Pharmacoeconomics of 3 Therapeutic Schemes for Chronic Pelvic Inflammation Disease. China Pharmacy, 17, 1557-1559.

[5] Yang, H.Y. (2007) Guizhi Fuling Capsule for the Treatment of Chronic Pelvic Inflammatory Disease, 100 Cases of Clinical Analysis. Chinese Journal of Misdiagnostics, 7, 1802-1803.

[6] Li, X.F., Cui, H. and Shan, S.L. (2008) The Clinical Observation of Microwave and Chinese Herb in the Treatment of Chronic Pelvic Inflammatory Disease. Chinese Community Doctors, 10,80 .

[7] Li, Y.J. (2009) The Clinical Observation of Integrated Traditional and Western Medicine in the Treatment of Chronic Pelvic Inflammatory Disease. Chinese Journal of Ethnomedicine and Ethnopharmacy, 18, 98.

[8] Wang, X.L. (2000) Combination of Traditional Chinese and Western Medicine in Treatment of Chronic Pelvic Inflammatory Mass 61 Cases. Traditional Chinese Medicinal Research, 13, 56 .

[9] Lu, Q. (2004) Combination Therapy for Chronic Pelvic Parametritis 66 Cases. Journal of Sichuan of Traditional Chinese Medicine, 22, 66-67.

[10] Chen, G.Z. (2005) A Changed Decoction of Gui Zhi Fu Ling Wan in Treating Chronic Pelvic Inflammatory Disease 40 Cases. Jilin Journal of Traditional Chinese Medicine, 25, 28.

[11] Zhang, H., Li, Y.B. and Jin, Z. (2009) Dang Gui Shao Yao San for the Treatment of Chronic Pelvic Inflammatory Disease, Clinical Observation of 30 Cases. Zhejiang Clinical Medical Journal, 11, 278-279.

[12] Zhang, H., Li, Y.B. and Jin, Z. (2009) The Curative Effect Observation with Dang Gui Shao Yao San for the Treatment of Chronic Pelvic Inflammatory Disease. Chinese Journal of Information on Traditional Chinese Medicine, 16, 58-59.

[13] Zhang, C.X. and Shi, H. (2006) Dang Gui Shao Yao San for the Treatment of Chronic Pelvic Inflammatory Disease, 43 Cases. Journal of Yangtze University, 3, 252-253.

[14] Lin, Y.Z. (2006) Theory and Clinical Research of Dang Gui Shao Yao San and Fumigation Lotion for the Treatment of Chronic Pelvic Inflammatory Disease. Beijing University of Chinese Medicine, Beijing.

[15] Ma, X.M. and Mu, Q.J. (2008) The Flavored Dang Gui Shao Yao San for the Treatment of Chronic Pelvic Inflammatory Disease, 30 Cases. China Practical Medical, 3, 134-135.

[16] Xu, H.M., Sun, X.W. and Wang, G.J. (1992) The Apply Hot Compress of Da Huang Mu Dan Tang for the Treatment of Chronic Pelvic Inflammatory Disease, 50 Cases. Hunan Journal of Traditional Chinese Medicine, 8, 47-48. 
[17] Zhang, L.N. (1999) Combined Internal and External Treatment on Pelvic Inflammatory Mass, 50 Cases. Henan Traditional Chinese Medicine, 19, 44-45.

[18] Wang, Y.X. (1999) The Curative Effect Observation of Da Huang Mu Dan Tang in Golden Chamber for the Treatment of Chronic Pelvic Inflammatory Disease. Chinese Journal of Information on Traditional Chinese Medicine, 6, 41-42.

[19] Hao, D.F. (2008) Combine Traditional Chinese with Western Medicine in Treatment of Chronic Pelvic Inflammatory Disease, the Curative Effect Observation of 53 Cases. Shanxi Journal of Traditional Chinese Medicine, 24, 29.

[20] Qin, F.S. and Dong, L. (2007) The Flavored Yi Yi Fu Zi Bai Jiang San for the Treatment of Chronic Pelvic Inflammatory Disease, 50 Cases. China’s Naturopathy, 15, 31-32.

[21] Zhang, T. (1993) Yi Yi Fu Zi Bai Jiang San for the Treatment of Chronic Pelvic Inflammatory Disease, 56 Cases. Shaanxi Journal of Traditional Chinese Medicine, 14, 533.

[22] Li, H.S. (1996) The Flavored Yi Yi Fu Zi Bai Jiang San for the Treatment of Pelvic Inflammatory Disease, 37 Cases. Zhejiang Journal of Traditional Chinese Medicine, 41, 300.

[23] Mou, C.L. (1997) The Flavored Yi Yi Fu Zi Bai Jiang San for the Treatment of Chronic Pelvic Inflammatory Disease, Clinical Observation on 67 Cases. China's Naturopathy, 5, 49.

[24] Shi, Y. (2001) Yi Yi Fu Zi Bai Jiang San for the Treatment of Chronic Pelvic Inflammatory Disease 98 Cases. Journal of Practical Traditional Chinese Medicine, 17, 14-15.

[25] Wei, X.F., Song, C.J. and Li, J. (2002) Combine Yi Yi Fu Zi Bai Jiang San with Dang Gui Shao Yao San for the Treatment of Chronic Pelvic Inflammatory Disease 62 Cases. Journal of Sichuan of Traditional Chinese Medicine, 20, 44.

[26] Shen, W.P. (2003) Fu Yan Mixture for the Treatment of Chronic Pelvic Inflammatory Disease 306 Cases. Traditional Chinese Medicinal Research, 16, 306.

[27] He, Y.F. (2005) Combine Traditional Chinese and Western Medicine in Treatment of Chronic Pelvic Inflammatory Disease 98 Cases. Shaanxi Journal of Traditional Chinese Medicine, 26, 1001-1002.

[28] Zhang, Y.J. (2006) The plus or minus of Yi Yi Fu Zi Bai Jiang San for the Treatment of Pelvic Inflammatory Mass 30 Cases. Zhejiang Journal of Traditional Chinese Medicine, 41, 339.

[29] Yu, J.C. and Zhao, J. (2008) The Flavored Yi Yi Fu Zi Bai Jiang San for the Treatment of Pelvic Inflammatory Mass 60 Cases. Journal of Practical Traditional Chinese Medicine, 24, 15.

\section{Submit or recommend next manuscript to SCIRP and we will provide best service for you:}

Accepting pre-submission inquiries through Email, Facebook, LinkedIn, Twitter, etc.

A wide selection of journals (inclusive of 9 subjects, more than 200 journals)

Providing 24-hour high-quality service

User-friendly online submission system

Fair and swift peer-review system

Efficient typesetting and proofreading procedure

Display of the result of downloads and visits, as well as the number of cited articles

Maximum dissemination of your research work

Submit your manuscript at: http://papersubmission.scirp.org/

Or contact ojog@scirp.org 